Patrícia Freitas Martins ${ }^{1}$

Carlito Lopes Nascimento Sobrinho

Marina Vieira Silva ${ }^{3}$

Nara Bernardes Pereira ${ }^{3}$

Cicília Marques Gonçalves ${ }^{3}$

Bárbara Santana Rebouças ${ }^{3}$

Luana de Assis Cartaxo ${ }^{3}$

\section{Afastamento por doença entre trabalhadores de saúde em um hospital público do estado da Bahia}

\author{
Sickness absenteeism among health care workers at a public \\ hospital in Bahia, Brazil
}

\author{
${ }^{1}$ Professora da Faculdade Nobre/FAN, \\ Feira de Santana, Bahia, Mestre em \\ Saúde Coletiva. \\ ${ }^{2}$ Professor Adjunto do Programa de \\ Pós-graduação em Saúde Coletiva, \\ Departamento de Saúde, Universidade \\ Estadual de Feira de Santana. \\ ${ }^{3}$ Estudantes de Medicina, Universidade \\ Estadual de Feira de Santana, Bolsistas \\ PROBIC.

\section{Contato:} \\ Patrícia Freitas Martins \\ Rua Belém, 680, casa B02, Conceição I \\ - Feira de Santana, Bahia \\ CEP: 44040-000 \\ E-mail: \\ ticamartinspf@yahoo.com.br
}

\section{Resumo}

Este trabalho descreveu as características sociodemográficas de trabalhadores de saúde, seu trabalho e a incidência de afastamento por doença de um hospital público do Estado da Bahia no período de $1^{\circ}$ de julho de 2005 a 30 de junho de 2006. Os dados foram coletados nas pastas cadastrais do setor de pessoal, nos atestados médicos de até 15 dias de afastamento e em documento da Secretaria Estadual de Saúde. Observou-se uma média de 2,31 atestados médicos por trabalhador. Dos 837 profissionais da equipe de saúde, 31,9\% afastaram-se por doença pelo menos uma vez no período. Destes, 91,0\% são do sexo feminino; $60,7 \%$ possuem idade $\geq 40$ anos; $60 \%$ possuem tempo de serviço na instituição $\geq 12,5$ anos; 70\% eram estatutários; 59,5\% tinham jornada de 40 horas semanais. A maioria dos afastados estava lotada na Emergência e na UTI e era do grupo de Enfermagem. Espera-se que este estudo fomente novas discussões sobre as características do trabalho em hospitais e estimule a implantação de serviço de atendimento à saúde do trabalhador no hospital estudado e a realização de novas investigações.

Palavras-chave: trabalhador de saúde, afastamentos por doença, hospital.

\begin{abstract}
This work describes the socio-demographic characteristics of health care workers, their work, and the incidence of sick leave in a public hospital in the State of Bahia, Brazil during the period between July 1, 2005 and June 30, 2006. Data was collected from the personnel department register, from doctors' statements for the leave, and from a document issued by the Bahia State Health Department. The results showed an average of 2.31 doctors' statements per worker. Among 837 workers, $31.9 \%$ were absent at least once due to illness, during the mentioned period. Of these, $91.0 \%$ were women; $60.7 \% \geq 40$ years old; $60 \%$ had been working at the institution for $\geq 12.5$ years; $70 \%$ were permanent civil servants covered by a specific pay system; 59.5\% worked 40 hours a week. Most of the absentees belonged to the nursing staff and worked either in the emergency ward or in the ICU. We expect that this study will encourage new discussions on the characteristics of hospital work, will incite the implementation of workers' health services in the hospital studied and new research on the issue.
\end{abstract}

Keywords: health care worker, sick leave, hospital. 


\section{Introdução}

Os profissionais da saúde formam um grupo especial de trabalhadores, com características próprias, como o predomínio do sexo feminino, divisão fragmentada de tarefas, rígida estrutura hierárquica e número quase sempre insuficiente de trabalhadores (REIS et al., 2003).

Nos hospitais públicos, os trabalhadores, em passado recente, eram contratados por concurso público. Atualmente, veem sendo admitidos mediante contratos por tempo determinado, o que dificulta a formação de quadros permanentes (NASCIMENTO SOBRINHO, 2005).

Segundo Gaspar (1997), os hospitais oferecem riscos para a saúde do trabalhador consideravelmente maiores que todas as outras unidades de saúde, pois apresentam piores condições de trabalho. Outros autores apontam que nos hospitais públicos, em virtude das inadequadas condições de funcionamento, essa situação se intensifica (MARZIALE; CARVALHO, 1998; PITTA, 2003).

Barboza e Soler (2003), Reis et al. (2003), dentre outros, ressaltam que o absenteísmo pode estar diretamente associado às condições de trabalho, cujas repercussões são visíveis na qualidade de vida, de saúde e de trabalho dos profissionais, e que é imprescindível a realização de estudos para analisar e prevenir o absenteísmo.

Segundo Reis et al. (2003), são incipientes as investigações sobre o absenteísmo por doença entre trabalhadores de saúde de hospitais públicos no Brasil. O estudo apresentado é importante por ser um dos primeiros da região do nordeste a estudar o absenteísmo por doença entre todas as categorias de trabalhadores de saúde dentro de hospital público.

O objetivo deste estudo foi descrever as características sociodemográficas, algumas características do trabalho e a incidência de afastamento por doença, por categoria profissional, dos trabalhadores da saúde de um hospital público do Estado da Bahia. Visa, ainda, estimular a reflexão sobre a necessidade de organização de serviços de saúde nos hospitais públicos voltados para prevenção de riscos, proteção e promoção da saúde dos trabalhadores da saúde.

\section{Metodologia}

Trata-se de um estudo epidemiológico descritivo. A coleta dos dados foi de fonte secundária, no setor de pessoal, utilizando uma ficha de coleta aplicada em: todas as pastas cadastrais dos profissionais de saúde afastados por doença; nos atestados médicos de até 15 dias, do período de $1^{\circ}$ de julho de 2005 a 30 de junho de 2006; e num documento da Secretaria de Saúde do
Estado da Bahia (SESAB), denominado ALFA, pois em muitas pastas cadastrais faltavam informações, como categoria profissional e tipo de contrato de trabalho.

As variáveis foram divididas em sociodemográficas: idade, sexo, situação conjugal e número de filhos; e ocupacionais: categoria profissional, setor de serviço, tempo de trabalho, carga horária e tipo de contrato de trabalho.

Os dados foram processados e analisados utilizando-se o programa SPSS for Windows 9.0, do Laboratório de Informática do Departamento de Saúde da Universidade Estadual de Feira de Santana (NORUŠIS, 1999). Foram calculadas as frequências absolutas e relativas das variáveis categóricas, a média e o desvio padrão das variáveis numéricas.

A coleta ocorreu no período de abril a agosto de 2007, sendo somente iniciada após autorização da direção do hospital e aprovação do Protocolo de Pesquisa pelo Comitê de Ética e Pesquisa - CEP/UEFS, conforme Ofício no 090/2007 de 28 de março de 2007, sob Protocolo no 035/2007 (CAAE - 0038.0.059.000-07).

\section{Resultados}

No período estudado, dos 837 profissionais da equipe de saúde do hospital, 31,9\% foram afastados do trabalho por doença pelo menos uma vez, representando uma elevada incidência de adoecimento. Obteve-se uma média de 2,31 atestados por trabalhador, com 2.746 dias de ausência no trabalho.

No grupo de enfermagem, composto por 517 profissionais, sendo 420 auxiliares de enfermagem (o setor de pessoal relacionou todos como auxiliar de enfermagem) e 97 enfermeiros, encontrou-se uma incidência de 41,2\% de afastamentos.

Quanto aos demais profissionais, 186 eram médicos, mas só 9 apresentaram atestados no período estudado; 44 eram técnicos de patologia clínica, aqui denominados como técnicos de laboratório; 20 técnicos de radiologia; 19 farmacêuticos; 19 fisioterapeutas; 10 odontólogos; 9 assistentes sociais; 9 nutricionistas; e 4 psicólogos. Como o número total desses profissionais era pequeno, não foi calculada a incidência de afastamento dos mesmos.

Dos 267 profissionais de saúde afastados por doença: $91,0 \%$ são do sexo feminino; $52,2 \%$ eram casados e $42,7 \%$ solteiros; $88,6 \%$ tinham pelo menos dois filhos; $60,7 \%$ apresentavam idade igual ou superior a 40 anos (Tabela 1).

A idade média foi de 41,6 \pm 9,7 anos, com mínima de 21 e máxima de 70 anos. Entre as mulheres, a média de idade foi de 41,0 \pm 9,7 anos, mínima de 21 e máxima de 70 anos e nos homens, a média de idade foi de 45,0 \pm 9,7 anos, mínima de 27 e máxima de 59 anos. 
Em relação à categoria profissional, o maior percentual de afastamento foi entre os auxiliares de enfermagem, com 64,0\%, seguidos pelos enfermeiros com 15,7\% e técnicos de laboratório com 7,1\%. Estes profissionais atuavam nos seguintes setores: Emergência, 30,0\%; Unidade de Tratamento Intensivo (UTI) - Adulto, 9,4\%; Laboratório, 6,7\% (Tabela 2). Destacaram-se ainda Ambulatório com 5,2\%, Clínica Cirúrgica com 5,6\% e Clínica Médica, Central Material de Esterilização (CME) e Centro Obstétrico (C.O.), todos três com o mesmo percentual de 4,5\% (Tabela 2).

Dos auxiliares de enfermagem afastados por doença, $34,5 \%$ atuavam na Emergência, $8,2 \%$ na UTI - Adulto e 7,0\% na CME. Entre os enfermeiros, 33,3\% atuavam na Emergência, 23,8\% na UTI - Adulto e 11,9\% na Clínica Cirúrgica. Dos técnicos de laboratório, $73,7 \%$ atuavam no Laboratório, 21,1\% no Banco de Sangue e 4,3\% na Central de Controle da Infecção Hospitalar - CCIH (Tabela 3).

Em relação à forma de contratação, 70,0\% dos profissionais tinham contrato de trabalho permanente (estatutário) e 30,0\% tinham contrato de trabalho temporário, denominado Regime de Direito Administrativo (REDA). Não foram encontrados profissionais cooperados afastados por doença. A carga horária de trabalho semanal mais frequente entre os profissionais de saúde afastados por doença foi de 40 horas semanais $(59,5 \%)$, seguidas por $30(36,0 \%)$ e 20 horas semanais $(4,5 \%)$ (Tabela 4$)$.

Com relação ao tempo de trabalho, observou-se que $60,0 \%$ tinham tempo de trabalho na instituição igual ou superior a 12,5 anos ( $\geq 150$ meses) e 40,0\% tinham menos de 12,5 anos (<150 meses) de serviço (Tabela 5). Estes resultados apontam que os profissionais afastados por doença, no hospital estudado, possuem um elevado tempo de serviço no hospital, com uma média de $12,7 \pm 8,9$ anos.

Entre os estatutários, 85,9\% apresentavam tempo de serviço igual ou superior a 12,5 anos ( $\geq 150$ meses), a média do tempo de serviço foi de 17,5 \pm 5,9 anos. Com relação à carga horária de trabalho, 85,0 \% apresentavam 40 horas semanais de trabalho (Tabela 5).

Entre os profissionais com contrato temporário afastados por doença, 100,0\% tinham tempo de serviço na instituição inferior a 12,5 anos, com média de 1,4 \pm 1,0 anos, porque o modelo de contrato utilizado tem prazo máximo de vigência de 4 anos; 95,0\% apresentavam jornada de trabalho semanal de 30 horas, conforme estabelecido no contrato adotado.

Tabela 1 Distribuição dos trabalhadores de um hospital público do Estado da Bahia, afastados por doença, entre jul/2005-jun/2006, segundo variáveis sociodemográficas

\begin{tabular}{lcc}
\hline \multicolumn{1}{c}{ Variável } & $N^{*}$ & $\%$ \\
\hline Sexo & 267 & 100,0 \\
Masculino & 24 & 9,0 \\
Feminino & 243 & 91,0 \\
Situação conjugal & 255 & 100,0 \\
Solteiro & 109 & 42,7 \\
Casado & 133 & 52,2 \\
Viúvo & 04 & 1,6 \\
Outros & 09 & 3,5 \\
Idade & 262 & 100,0 \\
$<40$ anos & 103 & 39,3 \\
$\geq 40$ anos & 159 & 60,7 \\
Número de filhos & 114 & 100,0 \\
$\leq 2$ & 101 & 88,6 \\
$>2$ & 13 & 11,4 \\
\hline
\end{tabular}

N* Resultados válidos, excluídas as perdas. 
Tabela 2 Categoria profissional e setor de trabalho dos trabalhadores de saúde afastados por doença em um hospital público do Estado da Bahia, jul/2005-jun/2006

\begin{tabular}{lcc}
\hline \multicolumn{1}{c}{ Variável } & $N^{*}$ & $\%$ \\
\hline Categoria profissional & 267 & 100,0 \\
Auxiliar de enfermagem & 171 & 64,0 \\
Enfermeiro & 42 & 15,7 \\
Técnico de laboratório & 19 & 7,1 \\
Médico & 9 & 3,4 \\
Fisioterapeuta & 8 & 3,0 \\
Outras & 18 & 6,8 \\
Setor de trabalho & 267 & 100,0 \\
Emergência & 80 & 30,0 \\
UTI- adulto & 25 & 9,4 \\
Laboratório & 18 & 6,7 \\
Clínica cirúrgica & 15 & 5,6 \\
Clínica médica & 12 & 4,5 \\
CME & 12 & 4,5 \\
C.O. & 12 & 4,5 \\
Outros & 93 & 34,8 \\
\hline
\end{tabular}

N* Resultados válidos, excluídas as perdas.

Tabela 3 Relação entre atividade profissional e setor onde atuavam os profissionais de saúde afastados por doença em um hospital público do Estado da Bahia, jul/2005-jun/2006

\begin{tabular}{lcccccc}
\hline $\begin{array}{l}\text { Setor de trabalho/Categoria } \\
\text { profissional }\end{array}$ & Auxiliar de enfermagem & \multicolumn{2}{c}{ Enfermeiro } & \multicolumn{2}{c}{ Técnico de laboratório } \\
& $N^{*}$ & $\%$ & $N^{*}$ & $\%$ & $N^{*}$ & $\%$ \\
\hline Emergência & 59 & 34,5 & 14 & 33,3 & - & - \\
UTI-adulto & 14 & 8,2 & 10 & 23,8 & - & - \\
Laboratório & - & - & - & - & 14 & 73,7 \\
Clínica cirúrgica & - & - & 5 & 11,9 & - & - \\
Clínica médica & - & - & - & - & - & - \\
CME & 12 & 7,0 & - & - & - & - \\
CCIH & - & - & - & - & 1 & 5,3 \\
Banco de sangue & - & - & - & - & 4 & 21,1 \\
Total & 171 & 100,0 & 42 & 100,0 & 19 & 100,0 \\
\hline
\end{tabular}

N* Resultados válidos, excluídas as perdas. 
Tabela 4 Características do trabalho, tipo de contrato, carga horária de trabalho e tempo de trabalho no hospital dos profissionais de saúde afastados por doença em um hospital público do Estado da Bahia, entre jul/2005-jun/2006

\begin{tabular}{ccc}
\hline \multicolumn{1}{c}{ Variável } & $N^{*}$ & $\%$ \\
\hline Tipo de contrato & 267 & 100,0 \\
Estável - estatutário & 187 & 70,0 \\
Temporário - REDA & 80 & 30,0 \\
Carga horária de trabalho & 267 & 100,0 \\
120 h mês/20 h semanais & 12 & 4,5 \\
180 h mês/30 h semanais & 96 & 36,0 \\
240 h mês/40 h semanais & 159 & 59,5 \\
Tempo de trabalho no hospital & 265 & 100,0 \\
$<12,5$ anos & 106 & 40,0 \\
$\geq 12,5$ anos & 159 & 60,0 \\
\hline
\end{tabular}

N* Resultados válidos, excluídas as perdas.

Tabela 5 Carga horária semanal e tempo de serviço dos profissionais afastados por doença em um hospital público do Estado da Bahia, jul/2005-jun/2006, segundo tipo de contrato

\begin{tabular}{lcccc}
\hline \multicolumn{1}{c}{ Tipo de contrato } & \multicolumn{2}{c}{ Estatutário } & \multicolumn{2}{c}{ Temporário } \\
\multicolumn{1}{l}{$N^{*}$} & $\%$ & $N^{*}$ & $\%$ \\
\hline Carga horária de trabalho & & & & \\
$\quad 120$ h mês/ 20 h semanais & 08 & 4,3 & 04 & 5,0 \\
180 h mês/30 h semanais & 20 & 10,7 & 76 & 95,0 \\
240 h mês/ 40 h semanais & 159 & 85,0 & - & - \\
Total & 187 & 100,0 & 80 & 100,0 \\
Tempo de trabalho no hospital & & & & \\
$<12,5$ anos & 26 & 14,1 & 80 & 100,0 \\
$\quad \geq 12,5$ anos & 159 & 85,9 & - & - \\
Total & 185 & 100,0 & 80 & 100,0 \\
\hline
\end{tabular}

N* Resultados válidos, excluídas as perdas.

\section{Discussão}

Barboza e Soler (2003), estudando afastamentos do trabalho por motivo de saúde entre profissionais de enfermagem, também encontraram o predomínio do sexo feminino, mas com idade entre 21 e 40 anos, situação também encontrada nos estudos de Pitta (2003) e Silva e Marziale (2000). Reis et al. (2003) e Raffone e Hennington (2005) encontraram resultados semelhantes, com predomínio do sexo feminino e idade igual e superior a 39 anos.

Andrade (1997) e Scavone (1997) apontam que o predomínio feminino entre as categorias profissionais estudadas ocorre porque as mulheres são as responsáveis pelos cuidados sanitários nos espaços domésticos e nas instituições de saúde.

A elevada incidência de afastamentos entre os trabalhadores da enfermagem provavelmente se deve ao fato de constituírem o maior número de trabalhadores e por serem um dos grupos mais submetidos às precárias condições de trabalho no setor de saúde, especialmente nos hospitais (BARBOZA; SOLER, 2003; REIS et al. 2003; SILVA; MARZIALE, 2000).

Os médicos, apesar da baixa ocorrência de afastamentos verificada, devem adoecer tanto quanto todos os demais trabalhadores de saúde. Pesquisas identifica- 
ram, por exemplo, elevada prevalência de Distúrbios Psíquicos Menores (DPM) nesta categoria (NASCIMENTO SOBRINHO et al., 2006). Entretanto, sabe-se que estes profissionais possuem maior liberdade de controle das suas atividades nas instituições públicas, a exemplo de faltarem ao serviço sem terem de justificar com um atestado médico.

Dentre os setores onde mais ocorreram os afastamentos, destacou-se a Emergência, local caracterizado por situações de estresse, convivência com dor, sofrimento, morte e acidentes. O setor caracteriza-se também por longas jornadas, plantões, trabalhos manuais pesados e repetitivos, falta de equipamentos, quadro reduzido de trabalhadores, dentre outros problemas, realidade também do hospital estudado (SATO, 2002; ROLLO, 2002).

Se a Emergência possui características difíceis de trabalho, estas se agravam para o grupo de enfermagem, principalmente os de nível médio, para os quais a sobrecarga física, a divisão fragmentada de tarefas e a rigidez da estrutura hierárquica é maior (BARBOZA; SOLER, 2003; FARIA; BARBOZA; DOMINGOS, 2005). A impossibilidade destes trabalhadores promoverem alterações na organização do trabalho, segundo Dejours (1992), constitui-se importante fator desencadeador de adoecimento.

O fato dos enfermeiros adoecerem nos mesmos setores dos auxiliares pode estar ligado ao fato de serem estes os responsáveis pela organização dos serviços, ou seja, à medida que os auxiliares faltam ao trabalho por adoecimento ou outro motivo, cabe ao enfermeiro reestruturar a equipe e garantir a qualidade da assistência, acumulando funções, o que a literatura aponta constituir fonte de adoecimento (BARBOZA; SOLER, 2003; REIS et al., 2003).

As UTI's também são identificadas como setores de elevado índice de adoecimento por terem elevada sobrecarga física e emocional e, sobretudo, pela alta exigência de qualificação profissional, pelo manuseio de equipamentos sofisticados, pelo acompanhamento ininterrupto dos pacientes, pelo ambiente, em geral, frio e isolado, sem contato com o restante da equipe e de acesso restrito aos familiares, que cobram mais cuidados desses profissionais (BARBOZA; SOLER, 2003; FARIA; BARBOZA; DOMINGOS, 2005).

Entre os auxiliares de enfermagem com idade inferior a 40 anos, houve um predomínio dos que atuavam nas Clínicas Cirúrgica, Médica e Materno-Infantil. Os auxiliares com idade igual e maior de 40 anos atuavam mais na Emergência e na UTI - Adulto, talvez porque estes setores necessitem de maior contingente de auxiliares de enfermagem e de maior qualificação profissional (BARBOZA; SOLER, 2003; SILVA; MARZIALE, 2000).

Entre os enfermeiros, observou-se que os de idade inferior a 40 anos atuavam predominantemente na UTI. Pode-se apontar que a especialização para o trabalho em Unidades de Terapia Intensiva é mais frequente en- tre os enfermeiros mais novos do que entre os que estão há mais tempo no mercado de trabalho, visto que esta é uma especialidade recente. As primeiras pós-graduações na área de enfermagem iniciaram-se em 1972, em São Paulo (DUCCI et al., 2007).

Encontrou-se o predomínio de contratos permanentes, similarmente ao encontrado nos estudos de Reis et al. (2003) e de Nascimento Sobrinho (2006). Outros estudos consultados não fizeram referência ao tipo de contrato, questão que merece maior investigação, pois a informalidade estimulada pelos setores privado e público vem favorecendo a desregulamentação dos direitos sociais e trabalhistas com potenciais efeitos sobre a saúde (ANTUNES, 2000; TAVARES, 2004).

Como em geral os funcionários permanentes são em maior número, é esperado um percentual maior de adoecimento entre os mesmos. Reis et al. (2003) argumentam que os estatutários, por serem permanentes e terem estabilidade, sentem-se mais seguros em se afastarem do trabalho. Contudo, aqueles autores admitem que o maior tempo de trabalho no hospital possa também ter contribuído para a redução da capacidade para o trabalho e para o adoecimento desses trabalhadores.

No que diz respeito à jornada de 40 horas semanais, os estudos apontam que, quanto maior as horas trabalhadas, maiores o desgaste e o adoecimento. Portanto, ao agregar uma intensa jornada às precárias condições de trabalho, típicas dos hospitais, aumentam-se as possibilidades de adoecimento entre os profissionais de saúde, conforme observado neste estudo e por outros autores (RAFFONE; HENNINGTON, 2005; NASCIMENTO SOBRINHO et al., 2006).

Em relação ao tempo de serviço, observa-se que esses profissionais estão envelhecendo no hospital, principalmente os permanentes. Segundo Raffone e Hennington (2005), considerando que o envelhecimento funcional é a perda da capacidade para o trabalho e que, em geral, começa a ser percebido antes do envelhecimento cronológico, ações de promoção da saúde podem diminuir ou até prevenir o adoecimento e a incapacidade, assim como a aposentadoria precoce.

\section{Considerações finais}

Os resultados encontrados neste estudo revelaram que os profissionais de saúde afastados por doença em um hospital público do Estado da Bahia, no período de $1^{\circ}$ de julho de 2005 a 30 de junho de 2006, são predominantemente do sexo feminino, com idade igual e superior a 40 anos, com tempo de serviço igual e superior a 12,5 anos. Associado a essas características estão o vínculo de trabalho estatutário e permanente e o predomínio da jornada de trabalho de 40 horas semanais.

Entre os setores de trabalho, destacaram-se a Emergência e a UTI como os locais onde atuava a maior parte 
dos trabalhadores afastados por doença. Com relação ao grupo profissional, encontrou-se predominância dos profissionais de Enfermagem. Os resultados apontaram, ainda, que o grupo de Enfermagem apresentou uma elevada incidência de afastamento por doença, dentre todos os grupos que atuavam no hospital estudado.

\section{Referências}

ANDRADE, M. L. A. G. A saúde no trabalho de homens e mulheres: uma visão de gênero. In: OLIVEIRA, E. M.; SCAVONE, L. (Org.). Trabalho, saúde e gênero na era da globalização. Goiânia: AB, 1997. p. 29-38.

ANTUNES, R. Adeus ao trabalho? Ensaio sobre as metamorfoses e a centralidade do mundo do trabalho. 7. ed. São Paulo: Cortez, 2000.

BARBOZA, D. B; SOLER, Z. A. S. G. Afastamentos do trabalho na enfermagem: ocorrências com trabalhadores de um hospital de ensino. Revista Latino-Americana de Enfermagem, Ribeirão Preto, v. 11, n. 2, p. 177-183, mar. 2003. Disponível em: <http://www.scielo.br/pdf/rlae/v11n2/v11n2a06.pdf>. Acesso em: 15 ago. 2006.

DEJOURS, C. A loucura do trabalho: estudo de psicopatologia do trabalho. 5. ed. São Paulo: Cortez, 1992.

DUCCI, A. J. et al. Produção científica brasileira de enfermagem em terapia intensiva de 1995 a 2004. Acta Paulista de Enfermagem, São Paulo, v. 20, n. 2, p. 216222, jun. 2007.

FARIA, A. F; BARBOZA, D. B.; DOMINGOS, N. A. M. Absenteísmo por transtornos mentais na enfermagem no período de 1995 a 2004. Arquivos de Ciências da Saúde, São José do Rio Preto, v. 12, n. 1, p. 14-20, jan./mar. 2005.

GASPAR, P. J. S. Enfermagem profissão de risco e de desgaste: perspectivas do enfermeiro do serviço de urgência. Lisboa, Nursing - ed. Portuguesa, v. 10, n. 109, p. 23-24, 1997.

GIATTI, L.; BARRETO, S. M. Situação do indivíduo no mercado de trabalho e iniqüidade em saúde no Brasil. Revista de Saúde Pública, São Paulo, v. 40, n. 1, p. 99106, jan./fev. 2006.

MARZIALE, M. H. P.; CARVALHO, E. C. Condições ergonômicas do trabalho da equipe de enfermagem em unidade de internação de cardiologia. Revista LatinoAmericana de Enfermagem, Ribeirão Preto, v. 6, n. 1, p. 99-117, jan. 1998.

MARZIALE, M. H. P.; ROBAZZI, M. L. C. C. O trabalho de enfermagem e a ergonomia. Revista LatinoAmericana de Enfermagem, Ribeirão Preto, v. 8, n. 6, p. 124-127, dez. 2000.
Os autores esperam que este trabalho possa fomentar novas investigações sobre as características e os riscos à saúde do trabalho em hospitais, especialmente na região nordeste do país. Espera-se ainda que o trabalho estimule a implantação de um serviço de atendimento médico à saúde do trabalhador no hospital estudado.
NASCIMENTO SOBRINHO, C. L. et al. Condições de trabalho e saúde dos médicos em Salvador, Brasil. Revista da Associação Médica Brasileira, São Paulo, v. 52, n. 2, p. 97-102, mar./abr. 2006.

NASCIMENTO SOBRINHO, C. L.; NASCIMENTO, M. A.; CARVALHO, F. M. Transformações no trabalho médico. Revista Brasileira de Educação Médica, Rio de Janeiro, v. 29, n. 2, p. 129-135, 2005.

NORUŠIS, M. J. SPSS 9.0 guide to data analysis. Englewood Cliffs: Prentice Hall, 1999.

PITTA, A. M. F. Hospital: dor e morte como ofício. 5. ed. São Paulo: Hucitec, 2003.

RAFFONE, A. M.; HENNINGTON, E. A. Avaliação da capacidade funcional dos trabalhadores de enfermagem. Revista de Saúde Pública, São Paulo, v. 39, n. 4, p. 669-676, ago. 2005.

REIS, R. J. et al. Fatores relacionados ao absenteísmo por doença em profissionais de enfermagem. Revista de Saúde Pública, São Paulo, v. 37, n. 5, p. 616-623, out. 2003.

ROLLO, A. A. É possível construir novas práticas assistenciais no hospital público? In: MERHY, E. E.; ONOCKO, R. (Org.). Agir em Saúde: um desafio para o público. São Paulo: Hucitec, 2002. p. 321-352.

SATO, L. Prevenção de agravos à saúde do trabalhador: replanejando o trabalho através das negociações cotidianas. Cadernos de Saúde Pública. Rio de Janeiro, v. 18, n. 5, p. 1147-1157, set./out. 2002.

SCAVONE, L. Saúde e gênero: impactos na família das doenças profissionais causadas pelo uso do amianto. In: OLIVEIRA, E. M.; SCAVONE, L. (Org.). Trabalho, saúde e gênero na era da globalização. Goiânia: AB, 1997. p. 39-50.

SILVA, D. M. P. P.; MARZIALE, M. H. P. Absenteísmo de trabalhadores de enfermagem em um hospital universitário. Revista Latino-Americana de Enfermagem, Ribeirão Preto, v. 8, n. 5, p. 44-51, out. 2000.

TAVARES, M. A. Os fios (in)visíveis da produção capitalista: informalidade e precarização do trabalho. São Paulo: Cortez, 2004. 